\title{
Hematoma Disecante de la Parte Torácica de la Aorta: Estudio Anatomopatológico de Siete Casos de Autopsias
}

\author{
Dissecting Hematoma of the Thoracic Aorta: Pathology Study of Seven Autopsy Cases \\ Julio César Guerini; Andrea Storniolo Squintone; Alba Borchert; \\ Ismael Bernardo Fonseca; Rodolfo Esteban Avila \& Luis Santos Spitale
}

\begin{abstract}
GUERINI, J. C.; STORNIOLO, S. A.; BORCHERT, A.; FONSECA, I. B.; AVILA, R. E. \& SPITALE, L. S. Hematoma disecante de la aorta torácica: estudio anatomopatológico de siete casos de autopsias. Int. J. Morphol., 29(4):1331-1334, 2011.

RESUMEN: El hematoma disecante de aorta (HDA) se define como la disección de la pared aórtica por la sangre, con formación de una nueva luz. Es la patología letal de la aorta más frecuentemente diagnosticada y ocurre con una frecuencia tres veces mayor que la rotura del aneurisma de la aorta abdominal. Nuestro objetivo es presentar siete casos de HDA observados en autopsias, describir los hallazgos anatomopatológicos y comentar la literatura. Los casos estudiados corresponden al Servicio de Patología del Instituto de Medicina Forense de Córdoba, Argentina. Los tejidos fueron fijados en formol al 10\%, incluidos en parafina y coloreados con hematoxilinaeosina, tricrómico de Masson y PAS (Peryodic Acid Schiff). De los siete casos presentados cuatro correspondieron a mujeres $(57,1 \%)$ y tres a hombres $(42,8 \%)$. Todos los casos estudiados tenían antecedentes de hipertensión arterial. La evolución posible de la disección aórtica incluye: rotura de la adventicia con hemorragia masiva y muerte, comunicación nuevamente con la luz aórtica, propagación al ostium coronario, isquemia de órganos y la formación de un aneurisma.
\end{abstract}

PALABRAS CLAVE: Hematoma; Disección; Aorta; Hipertensión arterial.

\section{INTRODUCCIÓN}

El hematoma disecante de aorta (HDA) se define como la disección de la pared aórtica por la sangre, con formación de una nueva luz.

Se acredita a Sennertus la primera descripción de esta entidad (Sennertus, 1650). Representó un diagnóstico postmortem hasta la primera parte del siglo XX (Maunoir, 1802). Es la patología letal de la aorta más frecuentemente diagnosticada y ocurre con una frecuencia tres veces mayor que la rotura del aneurisma de la parte abdominal de la aorta (Coady et al., 1999a). La prevalencia mundial estimada es de 0,5 a 2,95\% al año, siendo la incidencia de 2000 nuevos casos en el mismo período.

En una serie de autopsias, el diagnóstico pre-mortem fue realizado en el 15\% de los casos (Erbel et al., 2001). Nuestro objetivo es presentar siete casos de HDA observados en autopsias, describir los hallazgos anatomopatológicos y comentar la literatura.

\section{MATERIAL Y MÉTODO}

Los casos estudiados corresponden al Servicio de Patología del Instituto de Medicina Forense de Córdoba, Argentina.

La Tabla I resume los aspectos clínico-patológicos de los casos estudiados. Los tejidos fueron fijados en solución acuosa de formaldehído al 10\%, incluídos en parafina y coloreados con hematoxilina-eosina, tricrómico de Masson y PAS (Peryodic Acid Schiff).

\begin{tabular}{lccc}
\multicolumn{4}{l}{ Tabla I. Aspectos clínico-patológicos de los casos estudiados. } \\
\hline Caso & Protocolo & Sexo & Edad (años) \\
\hline 1 & A.P. 322-08 & Masculino & 46 \\
2 & A.P. 525-08 & Masculino & 74 \\
3 & A.P. 220-09 & Masculino & 41 \\
4 & A.P. 229-09 & Femenino & 77 \\
5 & A.P.339-09 & Femenino & 64 \\
6 & A.P.519-09 & Femenino & 41 \\
7 & A.P.661-10 & Femenino & 54 \\
\hline
\end{tabular}

Antecedentes clínicos:hipertensión arterial

II Cátedra de Patología y II Cátedra de Biología Celular, Histología y Embriología. Facultad de Ciencias Médicas. Universidad Nacional de Córdoba, Córdoba, Argentina. 


\section{RESULTADOS}

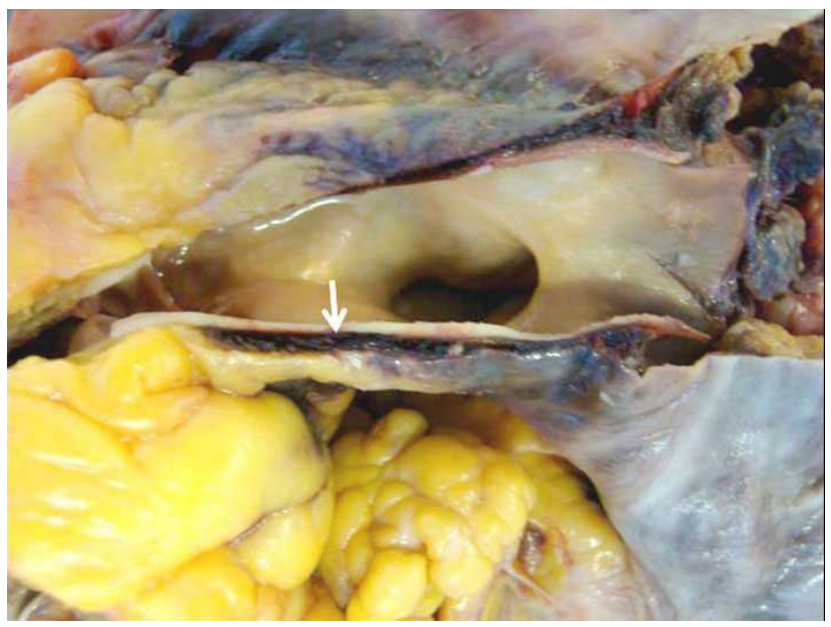

Fig. 1. Material hemático que diseca la túnica media o elástica y la adventicia de la aorta ascendente (flecha).

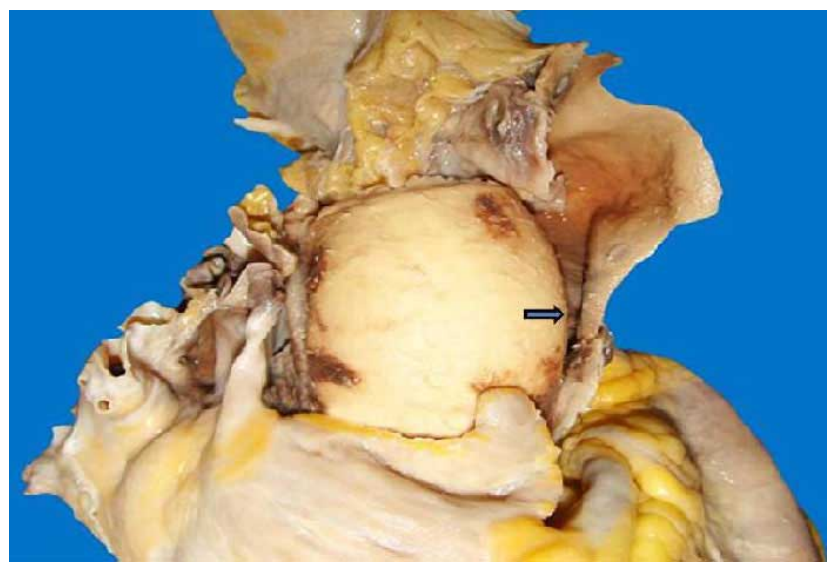

Fig. 2. Aorta ascendente que muestra la disección entre la túnica media o elástica y la adventicia (flecha).

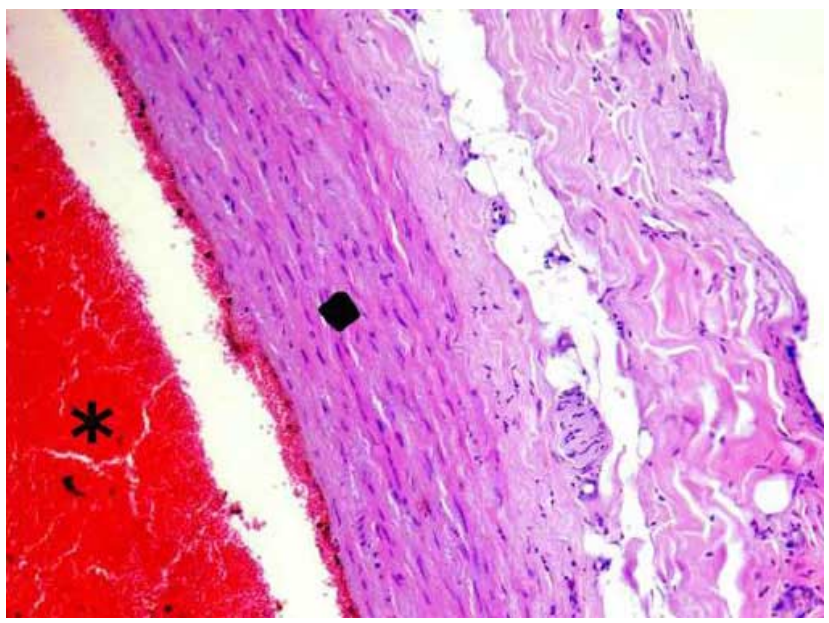

Fig. 3. Campo microscópico que muestra material hemático (asterisco) que diseca la íntima de la túnica elástica de la aorta (rombo). Coloración de H\&E 120 X.
De los siete casos presentados cuatro correspondieron a mujeres $(57,1 \%)$ y tres a hombres $(42,8 \%)$. El caso más joven tenía 41 años y el mayor 77 (edad media: 57 años).

Todos los casos estudiados tenían antecedentes de hipertensión arterial. El estudio anatomopatológico mostró disección hemática de las capas de la aorta ascendente y del cayado (Figs. 1 y 2), con infiltración hemática extensiva a la adventicia e inclusive al tejido adiposo en seis casos (Figs. 3 y 4). Asimismo, en cuatro casos $(57,1 \%)$ el hematoma disecante de la aorta comprometía a la arteria pulmonar (Figs. 5 y 6).

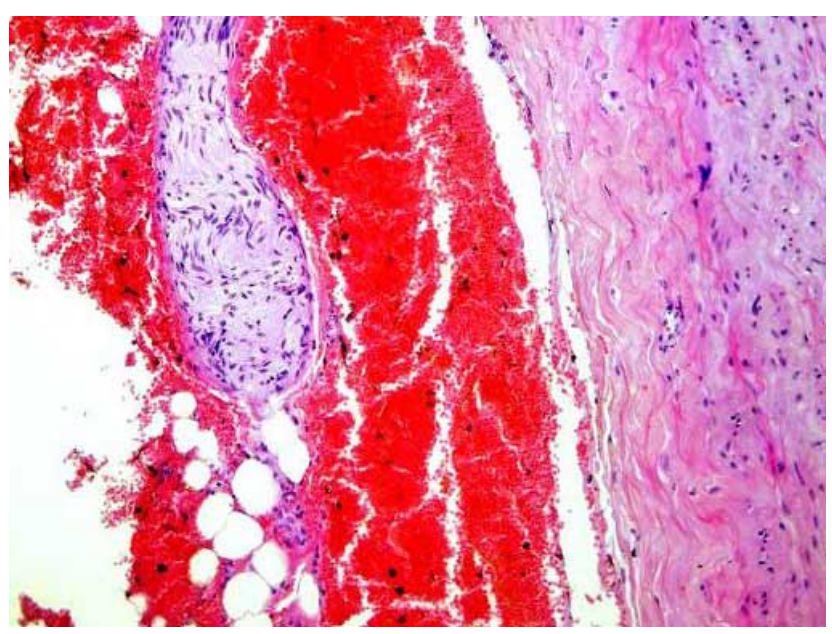

Fig. 4. Imagen microscópica con infiltración hemática de la aorta y extensión del proceso a la adventicia y tejido fibroadiposo. Coloración de H\&E 120 X.

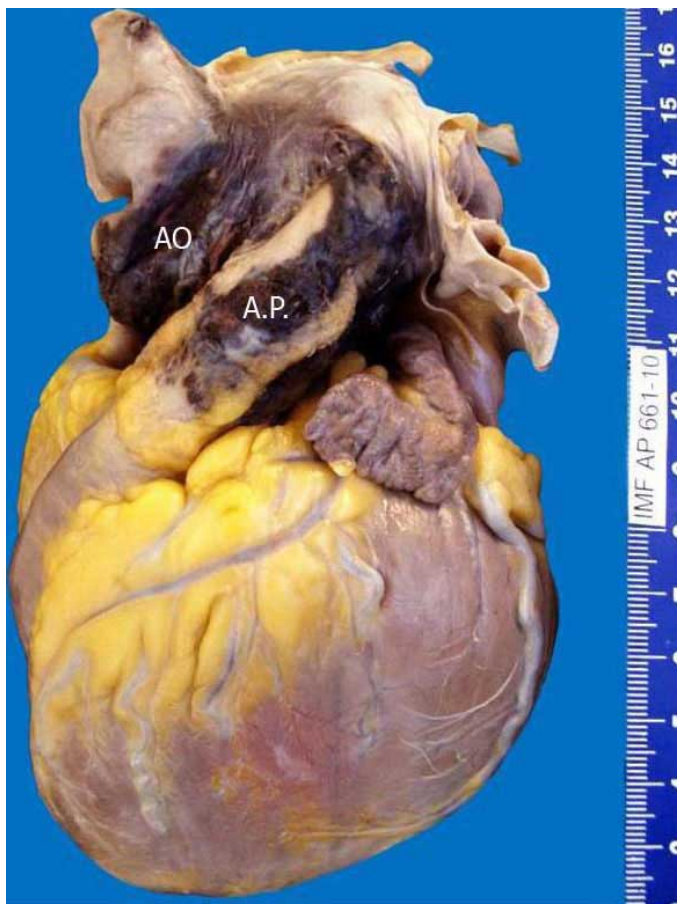

Fig. 5. Hematoma disecante que compromete la arteria pulmonar (AP) y la arteria aorta (AO). 


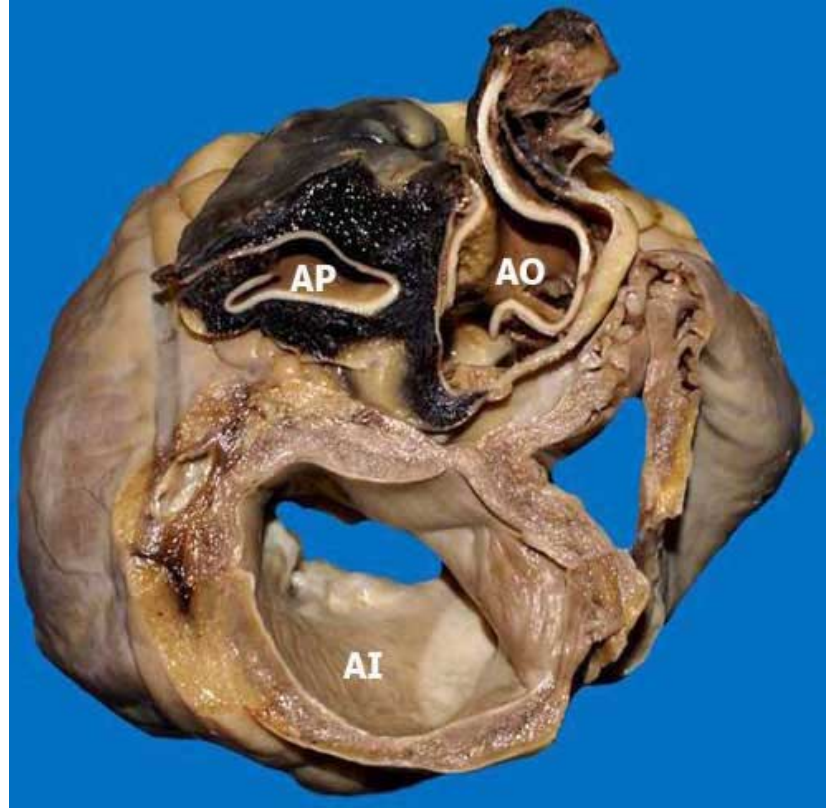

Fig. 6. Corte transversal del corazón a nivel auricular. Se observa la disección de la arteria aorta y de la arteria pulmonar. AO: Aorta. AP: Arteria pulmonar. AI: Aurícula izquierda

\section{DISCUSIÓN}

Existen varias hipótesis acerca de la etiología de la disrupción en la íntima (primera rotura), que permite al flujo de sangre crear un plano de clivaje dentro de la capa media de la pared aórtica. Entre dichas teorías se postulan alteraciones bioquímicas en el espesor de la capa media, fuerzas mecánicas (hipertensión arterial, hipervolemia, flujo sanguíneo turbulento, cateterización, etc.), degeneración o necrosis quística de la túnica media, úlceras ateroscleróticas penetrantes y hematoma intramural secundario a sangrado de los vasa vasorum, lo que produciría un aumento del "estrés" de la íntima durante la diástole, llevando a la rotura endotelial (Larson \& Edwards, 1984; Coady et al., 1999b).

En relación a la teoría de la degeneración quística de la media, la misma ha ido perdiendo vigencia con el paso de los años, debido a que se observa en un porcentaje bajo de pacientes, la mayoría de ellos niños.

El hematoma intramural se observa del 10 al $20 \%$ de los pacientes con disección aórtica aguda, lo que sugiere que podría ser un precursor (De Cecco et al., 2010).

La hipertensión arterial es la fuerza mecánica más frecuentemente asociada con la disección aórtica y se halla en más del 75\% de los casos. En nuestra serie de siete casos, todos tenían antecedentes de hipertensión arterial.
Otros factores de riesgo incluyen: enfermedades del tejido conectivo (Síndrome de Ehlers-Danlos, de Marfan, de Turner, etc.), aortitis, aneurisma aórtico torácico, valva aórtica bicúspide, fármacos, coartación de la aorta, embarazo, estenosis aórtica congénita, enfermedad renal poliquística, feocromocitoma, síndrome de Sheehan y síndrome de Cushing (Coady et al., 1999a.; Zinkernagel et al., 2005; Matura et al., 2007; Wakiyama et al., 2007; Reece et al., 2008; Tiryakioglu et al., 2009; Lentini et al., 2009).

Los dos sistemas de clasificación utilizados con mayor frecuencia en la práctica clínica son el de De Bakey (tipos I, II y III) y el de Stanford (tipos A y B) (Figs. 7 y 8). Ambos se basan en la localización y extensión de la disección, siendo el segundo un sistema funcional. A su vez, se pueden subclasificar según el tiempo de evolución en agudo (menos de dos semanas), subagudo (dos semanas a dos meses) y crónico (más de dos meses).
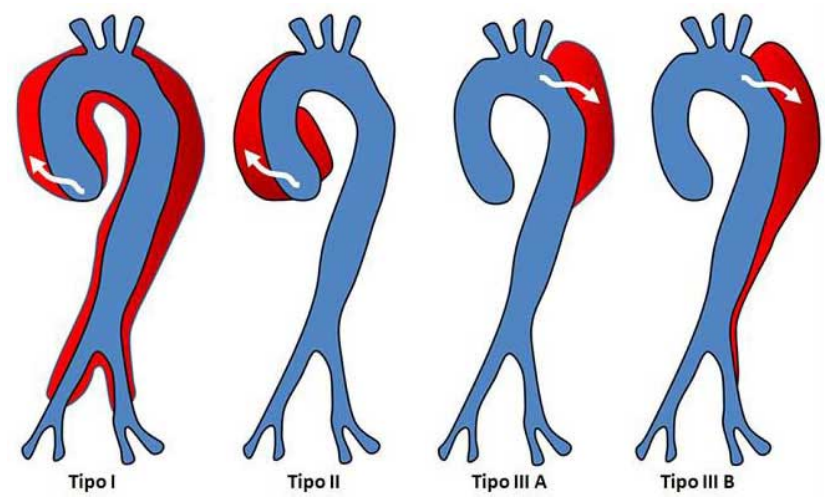

Fig. 7. Hematoma Disecante de Aorta. Clasificación de De Bakey.
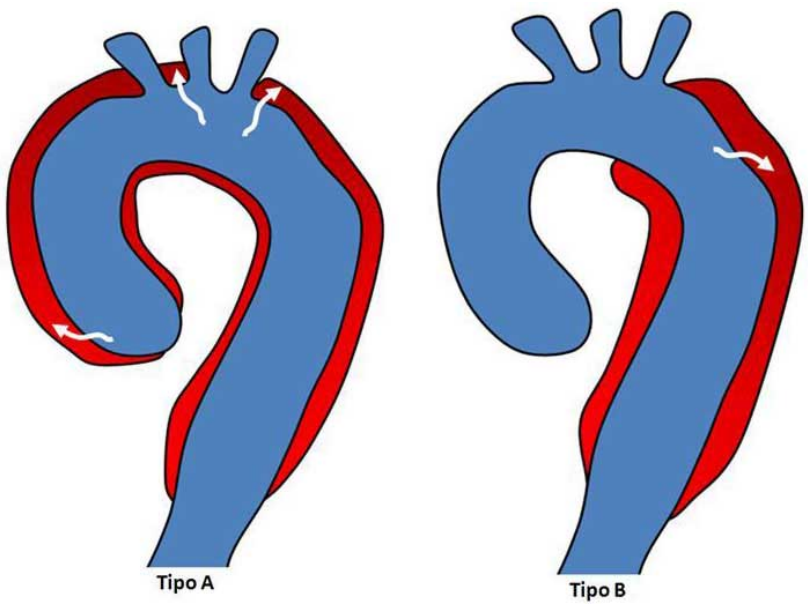

Fig. 8. Hematoma Disecante de Aorta. Clasificación de Stanford.

El tipo I (A) incluye disección que involucra la parte proximal de la aorta, arco aórtico y parte torácica de la aorta; el tipo II sólo incluye la parte ascendente de la aorta (englobado 
también en el tipo "A" de Stanford); el tipo III (B) se origina en la parte descendente de la parte torácica de la aorta y puede involucrar la parte abdominal de la aorta (Larson \& Edwards; Zinkernagel et al.; Matura et al.).

La lesión endotelial primaria generalmente es mayor al 50\% de la circunferencia de la aorta, pero la afección total de la misma es rara. La isquemia miocárdica y la rotura den- tro del pericardio, son las causas de muerte en el $80 \%$ de los casos de disección aguda.

La evolución posible de la disección aórtica incluye: rotura de la adventicia con la consecuente hemorragia masiva y muerte, comunicación nuevamente con la luz aórtica, propagación al ostium coronario, isquemia de órganos y la formación de un aneurisma.

GUERINI, J. C.; STORNIOLO, S. A.; BORCHERT, A.; FONSECA, I. B.; AVILA, R. E. \& SPITALE, L. S. Dissecting hematoma of the thoracic aorta: pathology study of seven autopsy cases. Int. J. Morphol., 29(4):1331-1334, 2011.

SUMMARY: Dissecting aortic hematoma (DAH) is defined as the dissection of the aortic wall by the blood, with formation of a new light. It is the deadliest disease of the aorta and occurs more frequently diagnosed at a rate three times greater than the ruptured aneurysm of the abdominal aorta. Our goal is to present seven cases of DAH observed in autopsies, describe the autopsy findings and comment on the literature. All cases studied belong to the Department of Pathology, Institute of Forensic Medicine of Cordoba, Argentina. The tissues were fixed in $10 \%$ formalin, embedded in paraffin and stained with hematoxylin-eosin, Masson trichrome and PAS (Peryodic Acid Schiff). Of all the cases studied, four were women (57.1\%) and three men (42.8\%). All had a history of hypertension. Evolution of aortic dissection may include: failure of the adventitia with massive hemorrhage and death, again communication with the aortic lumen, spread the coronary ostium, organ ischemia and aneurysm formation.

KEY WORDS: Hematoma; Dissection; Aorta; Hypertension.

\section{REFERENCIAS BIBLIOGRÁFICAS}

Coady, M. A.; Rizzo, J. A. \& Elefteriades, J. A. Pathologic variants of thoracic aortic dissections. Penetrating atherosclerotic ulcers and intramural hematomas. Cardiol Clin., 17(4):637-57, 1999a.

Coady, M. A.; Rizzo, J. A.; Goldstein, L. J. \& Elefteriades, J. A. Natural history, pathogenesis, and etiology of thoracic aortic aneurysms and dissections. Cardiol. Clin., 17(4):615-35; vii, 1999b.

De Cecco, C. N.; Di Girolamo, M.; Mancuso, F. \& David, V. Subacute intramural haematoma of the ascending aorta. Interact. Cardiovasc. Thorac. Surg., 11(5):701-2, 2010.

Erbel, R.; Alfonso, F.; Boileau, C.; Dirsch, O.; Eber, B.; Haverich, A.; Rakowski, H.; Struyven, J.; Radegran, K.; Sechtem, U.; Taylor, J.; Zollikofer, C.; Klein, W. W.; Mulder, B.; Providencia, L. A.; Task Force on Aortic Dissection \& European Society of Cardiology. Diagnosis and management of aortic dissection. Eur. Heart J., 22(18):1642-81, 2001.

Larson, E. W. \& Edwards, W. D. Risk factors for aortic dissection: a necropsy study of 161 cases. Am. J. Cardiol., 53(6):849-55, 1984.

Lentini, S.; Tancredi, F.; Benedetto, F. \& Gaeta, R. Type A aortic dissection involving the carotid arteries: carotid stenting during open aortic arch surgery. Interact. Cardiovasc. Thorac. Surg., 8(1):157-9, 2009.

Matura, L. A.; Ho, V. B.; Rosing, D. R. \& Bondy, C. A. Aortic dilatation and dissection in Turner syndrome. Circulation, 116(15):1663-70, 2007.
Maunoir, J. P. Mâemoires physiologiques et pratiques sur l'Anâevrisme et la ligature des artéres. Genáeve, Paschoud, 1802.

Reece, T. B.; Green, G. R. \& Kron, I. L. Aortic Dissection. In: Cohn, L. H. (Ed). Cardiac Surgery in the Adult. New York, McGraw-Hill, 2008. pp.1195-222.

Sennertus, D. Cap. 42. Op. Omn. Lib., 5:306, 1650.

Tiryakioglu, S. K.; Tiryakioglu, O.; Turan, T. \& Kumbay, E. Aortic dissection due to sildenafil abuse. Interact. Cardiovasc. Thorac. Surg., 9(1):141-3, 2009.

Wakiyama, H.; Nasu, M.; Fujiwara, H.; Kitamura, A. \& Okada, Y. Two surgical cases of acute aortic dissection in pregnancy with marfan syndrome. Asian Cardiovasc. Thorac. Ann., 15(5):e63$5,2007$.

Zinkernagel, M.; Wilhelm, M. J.; Tavakoli, R.; Turina, M. I. \& Genoni, M. Aortic dissection with potential origin from a mechanical bypass anastomosis. Ann. Thorac. Surg., 80(1):3168, 2005.

Dirección de correspondencia:

Ismael Bernardo Fonseca

II Cátedra de Patología y II Cátedra de Biología Celular, Histología y Embriología. Facultad de Ciencias Médicas

Universidad Nacional de Córdoba

Córdoba

ARGENTINA

Email: isma_fonseca@hotmail.com

Recibido : 08-06-2011

Aceptado: 05-09-2011 